PHYSICAL REVIEW E 95, 069904(E) (2017)

\title{
Erratum: Modeling rigid magnetically rotated microswimmers: Rotation axes, bistability, and controllability [Phys. Rev. E 90, 063006 (2014)]
}

Farshad Meshkati and Henry Chien Fu

(Received 8 June 2017; published 22 June 2017)

DOI: 10.1103/PhysRevE.95.069904

The rotational mobility in Eq. (38) has an error. It should read

$$
\mathbf{M}=\left(\begin{array}{ccc}
5.84 & 0 & 0 \\
0 & 8.96 & 0 \\
0 & 0 & 5.11
\end{array}\right) \times 10^{17} \mathrm{rad} \mathrm{N}^{-1} \mathrm{~s}^{-1} \mathrm{~m}^{-1}
$$

All the results and plots in the paper were obtained using this mobility matrix [Eq. (1)], not the one written in Eq. (38) of the original paper.

We thank Morozov et al. [1] for pointing out the error.

We are grateful to Kiarash Samsami and James D. Martindale for checking the statements in this Erratum. This work was funded by National Science Foundation Award No. CMMI-1650968 to H.C.F.

[1] K. I. Morozov, Y. Mirzae, O. Kenneth, and A. M. Leshansky, Phys. Rev. Fluids 2, 044202 (2017). 